\title{
PLANO DE RELUBRIFICAÇÃO DE UMA LINHA DE LAMINAÇÃO A FRIO*
}

\author{
Antônio Carlos Ataíde ${ }^{1}$ \\ Alan de Sousa Pereira² \\ Alysson Patrick Menezes Marques ${ }^{3}$
}

\section{Resumo}

O presente trabalho tem por objetivo revisar o atual plano de lubrificação de um laminador a frio modelo Sendzimir, tendo como foco principal determinar por meios analíticos, a confiabilidade dos processos de relubrificação à graxa de mancais de rolamento. Para a determinação dos parâmetros de relubrificação do restante dos conjuntos, que não são mancais de rolamento, foi realizada a consulta dos catálogos dos fabricantes para a determinação dos intervalos e quantidades, conforme recomendação destes. Para a realização deste trabalho, foi necessária uma extensa revisão bibliográfica, de normas e literaturas referentes a este tema. No final deste, objetiva-se a obtenção de um aumento da confiabilidade dos equipamentos, além de uma redução de custo com mão de obra e lubrificante durante os períodos de relubrificação.

Palavras-chave: Lubrificação; Relubrificação; Confiabilidade.

\section{PLAN OF RELUBRICATION OF A LINE OF COLD LAMINATION}

\begin{abstract}
This present project aims to review the current plan of lubrication of a cold rolling line. This project focused on determining, by analytical means, the reliability of the relubrication processes to bearing grease. For the determination of the relubrication parameters of the remaining assemblies, which are not rolling bearings, the manufacturer's catalogs were consulted for the determination of intervals and quantities, as recommended by them. For the accomplishment of this work, it was necessary an extensive bibliographical revision, of norms and literatures referring to this subject. At the end of this project, the objective is to obtain an increase in the reliability of the equipment, in addition to a reduction of cost with labor and waste of lubricating fluid during the periods of relubrication.
\end{abstract}

Keywords: Lubrication; Relubrication; Reliability.

1 Engenharia mecânica, Mestre em Engenharia Mecânica, Engenheiro Mecânico, Gerencia de Engenharia de Equipamentos, Aperam, Coronel Fabriciano, Minas Gerais, Brasil.

2 Estudante de Engenharia Mecânica, Bacharel, Estagiário, Manutenção, Aperam, Coronel Fabriciano, Minas Gerais, Brasil.

3 Estudante de Engenharia Mecânica, Bacharel, Estudante, Unileste, Coronel Fabriciano, Minas Gerais, Brasil. 


\section{INTRODUÇÃO}

A preocupação com a preservação dos instrumentos e ferramentas consta desde os indícios de formação de civilização. A partir de meados de 1950, surge na indústria Norte Americana o termo manutenção. Essa palavra foi substituída por conservação na França de maneira progressiva [1].

A globalização dos mercados é instalada tornando a concorrência industrial bastante acirrada, exigindo que esta aumente seu desempenho técnico e qualitativo, de modo a se fazerem competitivas em escala mundial [1].

Com a crescente evolução do mercado industrial foi necessário que as indústrias produzissem cada vez mais com menor custo e em menos tempo, para se obter um melhor desempenho. A busca pela redução de falhas passou a ser pauta nas pequenas e grandes empresas. No início esperava-se o equipamento falhar para gerar a manutenção, o que muitas vezes causava prejuízos incalculáveis, como acidentes com vítimas fatais, financeiros, como uma grande parada de um equipamento de vital importância para o processo produtivo.

A partir de estudos decorrentes na história, verificou-se que para o equipamento operar com a máxima eficiência possível, fazia-se necessário reduzir ao máximo as entropias presentes nos sistemas.

Com relação aos equipamentos industriais, o que gera maior perda de energia é a força de atrito, que é definida como a força que se opõe ao movimento de um corpo sobre o outro [2]. Mesmo antes do conhecimento profundo sobre tribologia (ciência que estuda 0 atrito) já temos grandes e concretos indícios da preocupação incessante sobre a redução dessa força [3]. Com a evolução dos estudos sobre o assunto verificou-se que existe dois tipos de atrito: atrito fluido e sólido, sendo que a energia necessária para realização do trabalho com 0 atrito fluido é consideravelmente menor em relação ao sólido [2]. Desde então passou a ser estudado os lubrificantes, pois a inserção de um filme fluido entre corpos diminui expressivamente o coeficiente de atrito.

Neste trabalho foi realizado um estudo relacionado a um plano de lubrificação de um laminador a frio modelo Sandzimir, com vista à elevação da confiabilidade, bem como uma redução de custo.

O trabalho (estudo) consistiu no levantamento de dados, tais como, carregamentos, contexto operacional, etc. de todos os conjuntos e subconjuntos, de forma a formular todo cálculo empírico a fim de ajustar a frequência de lubrificação e a qualidade da lubrificante.

\section{MATERIAIS E MÉTODOS}

O equipamento utilizado neste estudo foi um laminador de bobinas a frio modelo Sendzimir. Onde se objetivou realizar um estudo para a aplicação de uma nova metodologia de lubrificação, visando o aumento da confiabilidade e a redução de custos com lubrificantes.

Para a realização, foi analisada uma série de literaturas especializadas em relubrificação de mancais de rolamento.

Os conjuntos dos equipamentos analisados neste estudo foram: bobinadeiras de papel, desbobinadeira, bobinadeira, mancais moveis, acoplamentos, desempenadeiras, acionamento por eixos cardan e rolos puxadores.

De modo que a metodologia para o estudo destes pode ser vista na figura 1. 


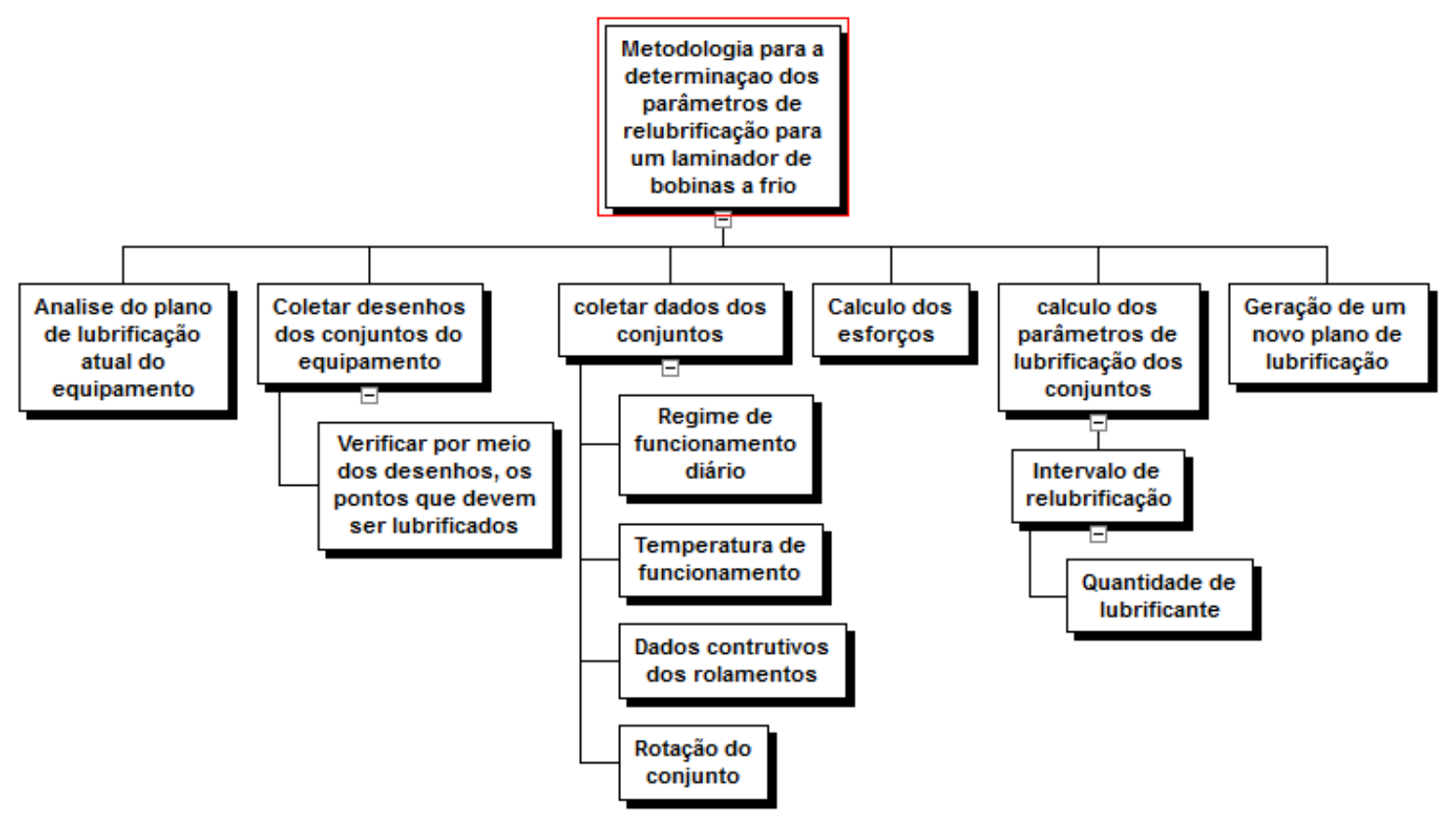

Figura 1. Metodologia de relubrificação.

Analisar o atual plano de lubrificação: A análise do atual plano de lubrificação consistiu em verificar principalmente, os intervalos de relubrificação para os conjuntos do laminador de bobinas, e as quantidades de graxa que eram aplicadas nestes intervalos;

Coletar desenhos dos conjuntos: A coleta de desenhos dos conjuntos teve vários objetivos, dentre eles:

- Localizar os pontos de lubrificação;

- Entender o funcionamento do equipamento;

- No caso dos rolamentos, verificar modelo e características;

- Analisar as dimensões do equipamento.

Características funcionais dos conjuntos: Para a determinação de um novo plano de lubrificação, é de suma importância analisar os seguintes pontos:

- Regime de trabalho do equipamento: $A$ análise do regime de trabalho do equipamento irá influenciar diretamente no intervalo de relubrificação dos conjuntos, uma vez que um equipamento trabalhando 3 horas por dia terá um intervalo de relubrificação superior de um mesmo equipamento trabalhando 24 horas por dia;

- Temperatura de funcionamento: Segundo [3] a viscosidade de um fluido lubrificante, é uma medida que varia diretamente com a temperatura, de modo que em ambientes com elevadas temperaturas acima de $75^{\circ} \mathrm{C}, 0$ intervalo de relubrificação tendera a diminuir;

Dados construtivos do rolamento: A Análise dos dados construtivos do rolamento tem como objetivo as seguintes verificações:

- Diâmetro externo, interno e largura do rolamento, para a determinação da quantidade de lubrificante a ser aplicada nos períodos de relubrificação;

- Análise da capacidade de carga dinâmica do rolamento;

- Análise da rotação máxima de trabalho do rolamento lubrificado a graxa. 
Calcular esforços atuantes: Se falando em mancais de rolamento, normalmente se consegue fazer o uso de vigas isostáticas ou hiperestáticas para o calculo das forças radiais e axiais atuantes sobre os rolamentos;

Determinação da quantidade e intervalos de relubrificação: Para a determinação dos intervalos de relubrificação dos conjuntos foi utilizada a (equação 1 ) .

Onde:

$$
T=K *\left(\frac{14 * 10^{6}}{n * d^{\frac{1}{2}}}-4 * d\right)
$$

$\mathrm{n}$ : Rotação do rolamento;

d: Diâmetro interno do rolamento;

$\mathrm{K}$ : Fator de segurança;

T: Período de relubrificação em horas.

Onde o fator de segurança $\mathrm{K}$ poderá ser determinado em função da (equação 2) :

$K=F 1 * F 2 * F 3 * F 4 * F 5 * F 6 * F 7$

De modo que os parâmetros de F1 a F7 podem ser determinado com base na tabela 1:

Tabela 1. Fatores de segurança para determinação dos intervalos de relubrificação

\begin{tabular}{|c|c|c|}
\hline \multicolumn{3}{|c|}{ Parâmetros de correção do período de relubrificação } \\
\hline \multirow{3}{*}{ Influência da poeira e umidade } & Média & $\mathrm{F} 1=0,7$ a 0,9 \\
\hline & Forte & $\mathrm{F} 1=0,4$ a 0,7 \\
\hline & Muito Forte & $\mathrm{F} 1=0,1$ a 0,4 \\
\hline \multirow{3}{*}{ Influência da vibração } & Média & $\mathrm{F} 2=0,7$ a 0,9 \\
\hline & Forte & $\mathrm{F} 2=0,4$ a 0,7 \\
\hline & Muito Forte & $\mathrm{F} 2=0,1$ a 0,4 \\
\hline \multirow{3}{*}{ Influência da temperatura } & Média (até $75^{\circ} \mathrm{C}$ ) & $\mathrm{F} 3=0,7$ a 0,9 \\
\hline & Forte $\left(\right.$ de $75^{\circ} \mathrm{C}$ a $\left.85^{\circ} \mathrm{C}\right)$ & $\mathrm{F} 3=0,4$ a 0,7 \\
\hline & Muito Forte (acima de $85^{\circ} \mathrm{C}$ ) & $\mathrm{F} 3=0,1$ a 0,4 \\
\hline \multirow{3}{*}{ Influência da relação de carga } & $\mathrm{P} / \mathrm{C}=0,1$ a 0,15 & $\mathrm{~F} 4=0,7$ a 0,9 \\
\hline & $\mathrm{P} / \mathrm{C}=0,15$ a 0,25 & $\mathrm{~F} 4=0,4$ a 0,7 \\
\hline & $\mathrm{P} / \mathrm{C}=0,25$ a 0,35 & $\mathrm{~F} 4=0,1$ a 0,4 \\
\hline \multirow{3}{*}{$\begin{array}{l}\text { Influência da forma construtiva do } \\
\text { rolamento }\end{array}$} & $\begin{array}{l}\text { Rolamentos de esferas, } \\
\text { autocompensadores e de contato } \\
\text { angular }\end{array}$ & $\mathrm{F} 5=1$ a 1,6 \\
\hline & $\begin{array}{l}\text { Rolamentos de rolos cilíndricos, de } \\
\text { agulhas e cônicos }\end{array}$ & $\mathrm{F} 5=3$ a 4 \\
\hline & Rolamentos axiais & $\mathrm{F} 5=5 \mathrm{a} 6$ \\
\hline \multirow{3}{*}{$\begin{array}{l}\text { Influência do fluxo de ar através do } \\
\text { rolamento }\end{array}$} & Sem fluxo & $\mathrm{F} 6=0,7$ a 0,9 \\
\hline & Pouco fluxo & $\mathrm{F} 6=0,5$ a 0,7 \\
\hline & Fluxo forte & $\mathrm{F} 6=0,1$ a 0,5 \\
\hline \multirow{3}{*}{$\begin{array}{l}\text { Influência da perca proveniente de } \\
\text { defeitos advindos de vedações }\end{array}$} & $\mathrm{n} / \mathrm{N}=0,1$ a 0,15 & $\mathrm{~F} 4=0,7$ a 0,9 \\
\hline & $\mathrm{n} / \mathrm{N}=0,15$ a 0,25 & $\mathrm{~F} 4=0,4$ a 0,7 \\
\hline & $\mathrm{n} / \mathrm{N}=0,25$ a 0,35 & $\mathrm{~F} 4=0,1$ a 0,4 \\
\hline
\end{tabular}

Na tabela 1 as variáveis apresentadas são:

N: Rotação máxima do rolamento;

P: Carga dinâmica equivalente aplicada sobre o rolamento;

C: Capacidade de carga dinâmica do rolamento.

Para a determinação da quantidade de lubrificante a ser aplicado nos períodos de relubrificação deve se fazer uso da (equação 3 ):

Onde:

$$
G=0,005 * D * B
$$


$\mathrm{G}=$ quantidade de lubrificante em gramas;

$\mathrm{D}=$ Diâmetro externo do rolamento;

$\mathrm{B}=$ Largura do rolamento.

A determinação dos parâmetros de relubrificação de todos os conjuntos seguiu a mesma metodologia.

Gerar novo plano de relubrificação: Uma vez determinado o intervalo e a quantidade de lubrificante a serem aplicados nos períodos de relubrificação, pode ser gerado um novo plano de relubrificação que seja confiável e seguro.

\subsection{EXEMPLO DE APLICAÇÃO DESTA METODOLOGIA}

Como exemplo de aplicação da metodologia, será apresentado abaixo a aplicação em um conjunto do laminador, que é a desempenadeira.

A desempenadeira é um equipamento mecânico que tem como função de desempenar as pontas inicial e final da bobina de aço. A figura 2 mostra a vista geral da desempenadeira.

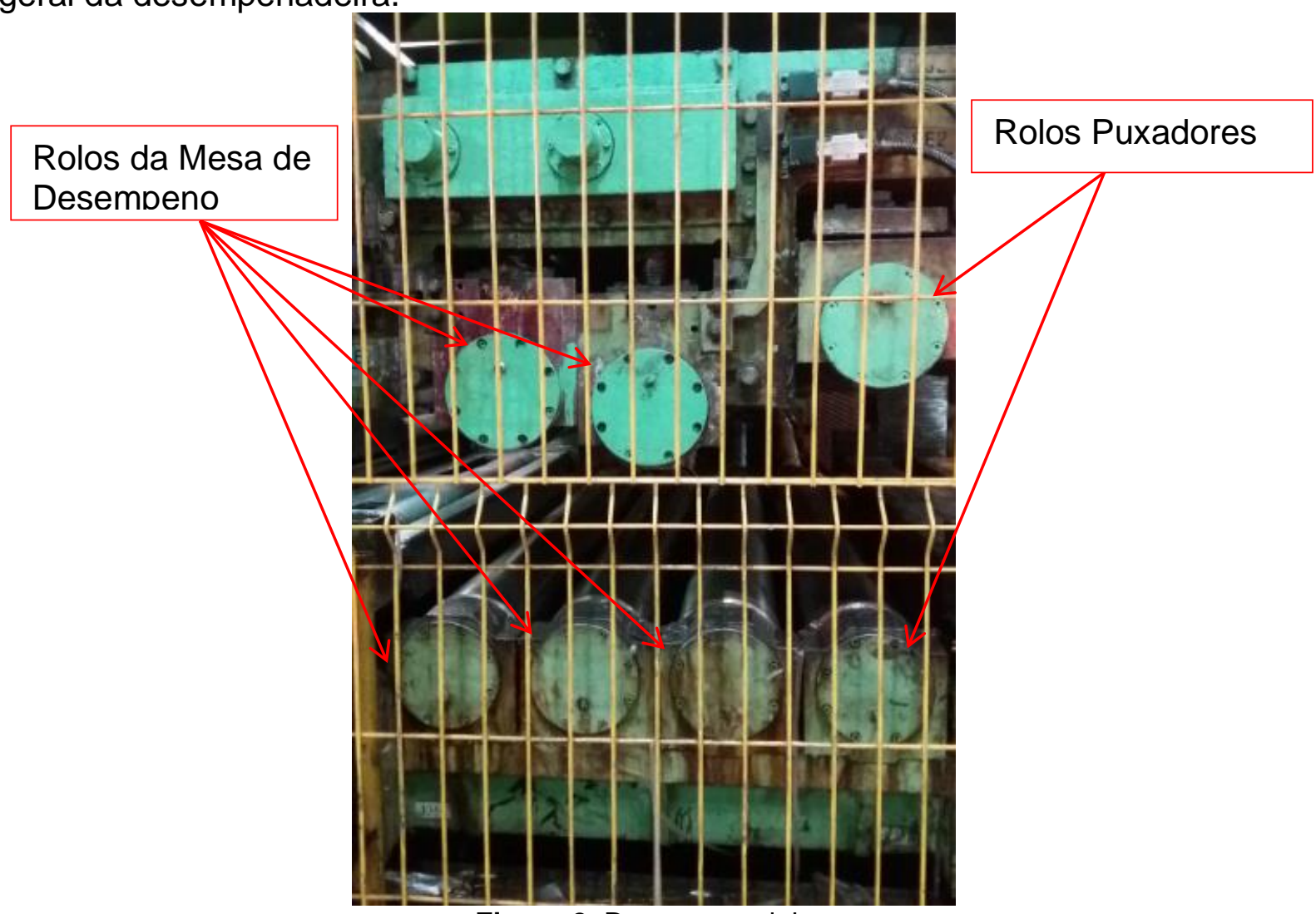

Figura 2. Desempenadeira.

A desempenadeira possui o plano relubrificação, conforme pode ser visto na tabela 2: 
Tabela 2. Atual plano de relubrificação da desempenadeira

\begin{tabular}{|l|l|l|l|}
\hline \multicolumn{4}{|c|}{ Atual plano de lubrificação da Desempenadeira } \\
\hline Atividade & Pontos & Quantidade & Intervalo \\
\hline Relubrificação dos & Acionamento por cardan & 30 gramas & 84 dias \\
$\begin{array}{l}\text { Relubrificação puxadores } \\
\text { mancais de rolamento }\end{array}$ & $\begin{array}{l}\text { Rolos gramas } \\
\text { superiores e inferiores }\end{array}$ & 84 dias \\
\hline $\begin{array}{l}\text { Relubrificação dos } \\
\text { mancais de rolamento }\end{array}$ & $\begin{array}{l}\text { Rolos da mesa de de } \\
\text { desempeno }\end{array}$ & 130 gramas & 84 dias \\
\hline Relubrificação & Guias deslizantes & 10 gramas & 84 dias \\
\hline
\end{tabular}

\subsubsection{PROPOSTA DE PLANO DE RELUBRIFICAÇÃo PARA A} DESEMPENADEIRA

Para a determinação de um plano de relubrificação mais eficiente e confiável, foi utilizada a metodologia conforme pode ser vista na figura 1 , de modo que os seus respectivos desenhos foram analisados para verificar a localização de todos os pontos de lubrificação, e para verificar nos caso dos mancais de rolamento, qual a forma construtiva destes.

Onde nos casos dos mancais de rolamento, verificou se conforme a tabela 3 as características construtivas dos rolamentos da desempenadeira:

Tabela 3. Características dos rolamentos da desempenadeira

\begin{tabular}{|c|c|}
\hline \multicolumn{2}{|c|}{$\begin{array}{c}\text { CARACTERÍSTICAS DOS ROLAMENTOS DA } \\
\text { DESEMPENADEIRA }\end{array}$} \\
\hline Tipo de Rolamento & $\begin{array}{c}\text { SKF } \\
22217 \mathrm{E}\end{array}$ \\
\hline $\begin{array}{c}\text { Capacidade de Carga } \\
\text { Dinâmica (C) }\end{array}$ & $291 \mathrm{KN}$ \\
\hline $\begin{array}{c}\text { Rotação Máxima do } \\
\text { Rolamento }\end{array}$ & $5600 \mathrm{RPM}$ \\
\hline $\begin{array}{c}\text { Largura do Rolamento (B) } \\
\text { Diâmetro Externo do } \\
\text { Rolamento }\end{array}$ & $36 \mathrm{~mm}$ \\
\hline $\begin{array}{c}\text { Diâmetro Interno do } \\
\text { Rolamento }\end{array}$ & $150 \mathrm{~mm}$ \\
\hline
\end{tabular}

As características de trabalho deste conjunto podem ser observadas na tabela 4: 
Tabela 4. Características de trabalho destes rolamentos

\begin{tabular}{|c|c|}
\hline \multicolumn{2}{|c|}{ CARACTERÍSTICAS DE TRABALHO } \\
\hline Regime de trabalho diário & 3 horas \\
\hline Rotação de trabalho & 36 RPM \\
\hline
\end{tabular}

O rolo puxador da desempenadeira é deslocado verticalmente por um cilindro com uma unidade de alimentação com pressão de $60 \mathrm{Kgf} / \mathrm{cm}^{2}$, enquanto os rolos da mesa de desempeno são também submetidos por um cilindro com pressão de 30 $\mathrm{Kgf} / \mathrm{cm}^{2}$.

De modo que para se determinar a carga aplicada sobre os rolamentos dos puxadores e da mesa de desempeno, deve se calcular a força que o cilindro faz sobre os rolos. Sabendo que para determinar a pressão pode-se utilizar a equação 4:

Podendo assim determinar a força por meio da (equação 5):

$$
P=\frac{F}{A}
$$

Onde:

$$
F=P * A
$$

F: Força exercida pelo cilindro;

A: Área do êmbolo do cilindro;

P: Pressão do cilindro.

A tabela 5 apresenta as cargas aplicadas sobre os rolos puxadores e sobre os rolos da mesa de desempeno:

Tabela 5. Características dos cilindros

\begin{tabular}{|l|l|l|l|l|}
\hline \multicolumn{5}{|c|}{ Características dos cilindros } \\
\hline & $\begin{array}{l}\text { Piâmetro do } \\
\text { êmbolo }\end{array}$ & Área & Força \\
\hline Rolos Puxadores & $\begin{array}{l}60 \\
\mathrm{Kgf} / \mathrm{cm}^{2}\end{array}$ & $140 \mathrm{~mm}$ & $\begin{array}{l}15393,8 \\
\mathrm{~mm}^{2}\end{array}$ & $90,6 \mathrm{KN}$ \\
\hline Rolos da mesa de desempeno & $\begin{array}{l}35 \\
\mathrm{Kgf} / \mathrm{cm}^{2}\end{array}$ & $280 \mathrm{~mm}$ & $\begin{array}{l}61575,2 \\
\mathrm{~mm}^{2}\end{array}$ & $211,4 \mathrm{KN}$ \\
\hline
\end{tabular}

Tendo as forças aplicadas sobre os rolos, foi possível determinar por meio de vigas isostáticas os esforços atuantes nos rolamentos.

Logo com base na figura 3, pode-se observar as reações de apoio atuando sobre os rolos puxadores: 


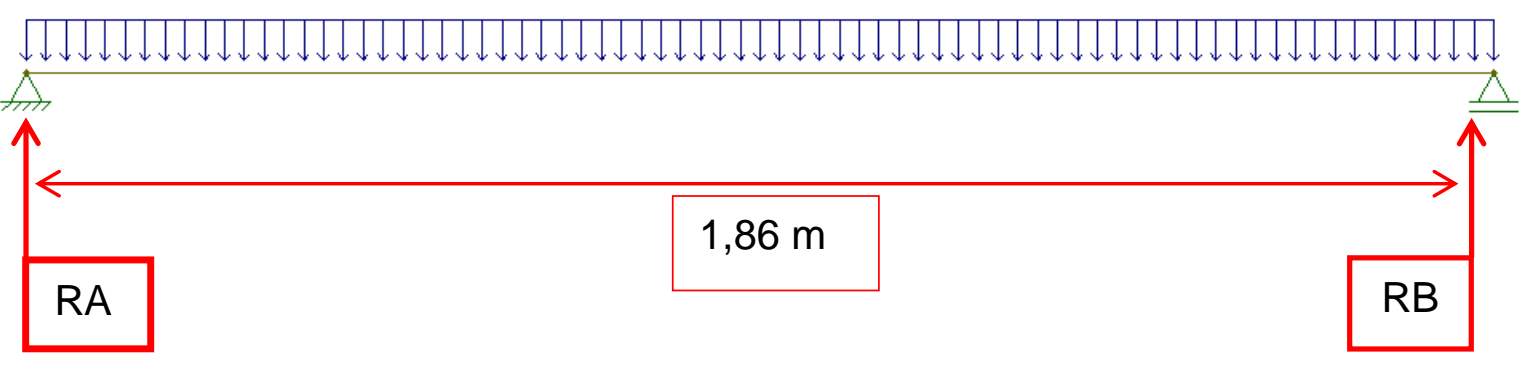

Figura 3. Esforços atuando nos rolamentos dos rolos puxadores

De modo que para se determinar RA e RB, deve se fazer uso das equações de equilíbrio:

$$
\begin{array}{r}
\Sigma F y=0 \\
R A+R B=50,98 K N \\
\Sigma M B=0 \\
-R A * 1,86 m+90,6 K N * 0,93 m=0 \\
R A=47,4 K N \\
R B=47,4 K N
\end{array}
$$

Para a determinação da carga atuante sobre os rolos da mesa de desempeno, deve se dividir a carga encontrada na tabela 5 por dois, uma vez que essa força atua de forma distribuída sobre dois cilindros.

Podendo assim determinar a carga atuante sobre os rolamentos da mesa da desempenadeira, também considerando esta como uma viga isostática, conforme pode ser visto na figura 4 :

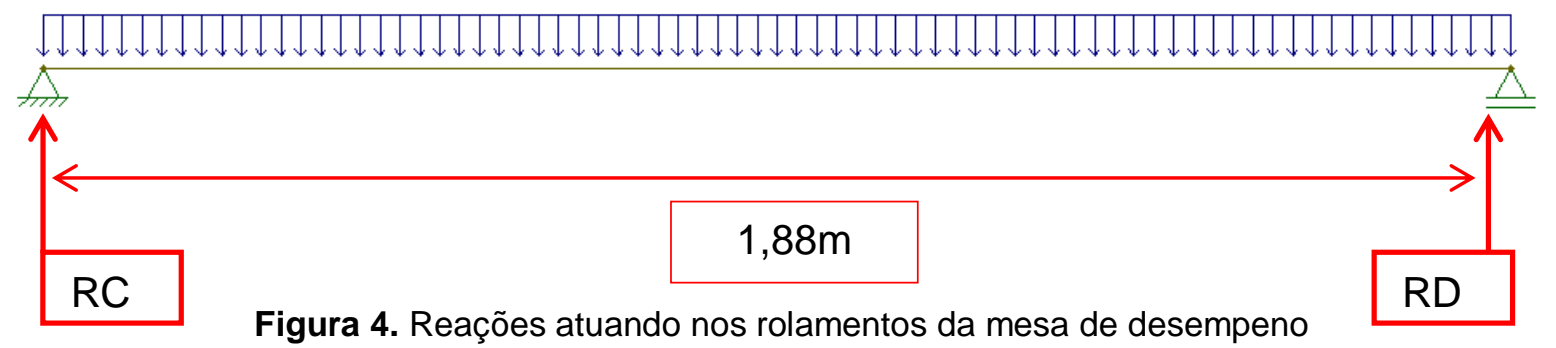

De modo que para se determinar $\mathrm{RC}$ e $\mathrm{RD}$, também por meio das reações de equilíbrio:

$$
\begin{array}{r}
\boldsymbol{\Sigma} \boldsymbol{F y}=\mathbf{0} \\
R C+R D=105,7 K N \\
\Sigma M B=0 \\
-R C * 1,86 m+90,6 K N * 0,93 m=0 \\
R C=52,8 K N \\
R D=52,8 K N
\end{array}
$$

Tendo-se determinado as reações atuantes sobre os mancais de rolamento, é possível determinar os parâmetros de relubrificação para os rolos da mesa de desempeno e para os rolos puxadores.

A Determinação do fator de segurança $\mathrm{K}$ para os rolos pode ser vista na tabela 6 : 
Tabela 6. Determinação do fator de segurança $K$

\begin{tabular}{|c|c|c|}
\hline \multicolumn{3}{|c|}{ Determinação do fator de segurança K } \\
\hline & Mesa de desempeno & Rolos puxadores \\
\hline F1 & 0,7 & 0,7 \\
\hline F2 & 0,7 & 0,7 \\
\hline F3 & 0,7 & 0,7 \\
\hline F4 & 0,2 & 0,2 \\
\hline F5 & 1,1 & 1,1 \\
\hline F6 & 0,7 & 0,7 \\
\hline F7 & 0,7 & 0,7 \\
\hline & $\mathrm{K}=0,0369$ & $\mathrm{~K}=0,0369$ \\
\hline
\end{tabular}

Tendo-se determinado o fator de segurança $K$ para ambos os rolos, torna se possível a determinação do intervalo de relubrificação.

Determinação do intervalo de relubrificação para os rolos puxadores:

Determinação do intervalo de relubrificação para a mesa de desempeno:

$$
T=0,0369 *\left(\frac{14 * 10^{6}}{36 * 85^{\frac{1}{2}}}-4 * 85\right)
$$

$$
T=0,0369 *\left(\frac{14 * 10^{6}}{36 * 85^{\frac{1}{2}}}-4 * 85\right)
$$

A quantidade poderá ser determinada por meio da equação 3 , onde esta quantidade será a mesma para ambos os rolos:

$$
\begin{array}{r}
G=0,005 * 36 * 150 \\
G=32 \text { gramas. }
\end{array}
$$

O eixo cardan conforme recomendação dos fabricantes poderá ter sua relubrificação com intervalo de aproximadamente 180 dias.

\section{RESULTADOS E disCUSSÃo}

Para a realização do plano do restante dos conjuntos do laminador de bobinas, a metodologia utilizada foi a mesma da desempenadeira, na qual foi alvo de exemplo anteriormente.

A tabela 7 nos revela um comparativo entre a quantidade de lubrificante utilizada, no plano original e no plano proposto. 
Tabela 7.Comparação entre as quantidades de lubrificante utilizadas nos conjuntos do Laminador anualmente

\begin{tabular}{|c|c|c|c|c|c|}
\hline \multicolumn{6}{|c|}{ Comparativo da quantidade de lubrificante utilizado nos conjunto em gramas } \\
\hline & & \multicolumn{2}{|c|}{ Antes } & \multicolumn{2}{|c|}{ Depois } \\
\hline & & $\begin{array}{l}\text { Frequência } \\
\text { Anual }\end{array}$ & $\begin{array}{c}\text { Quantidade } \\
\text { (Gramas) }\end{array}$ & $\begin{array}{l}\text { Frequência } \\
\text { Anual }\end{array}$ & $\begin{array}{c}\text { Quantidade } \\
\text { (Gramas) }\end{array}$ \\
\hline \multirow[t]{2}{*}{$\begin{array}{l}\text { Bobinadeira } \\
\text { Direita }\end{array}$} & $\begin{array}{c}\text { Rolo } \\
\text { Prendedor }\end{array}$ & 4 & 40 & 2 & 34 \\
\hline & Ponta Rotativa & 4 & 1000 & 4 & 413 \\
\hline \multirow[t]{2}{*}{$\begin{array}{l}\text { Bobinadeira } \\
\text { Esquerda }\end{array}$} & $\begin{array}{c}\text { Rolo } \\
\text { Prendedor }\end{array}$ & 4 & 40 & 2 & 34 \\
\hline & Ponta Rotativa & 4 & 1000 & 4 & 413 \\
\hline \multirow[t]{2}{*}{ Desbobinadeira } & $\begin{array}{c}\text { Rolo } \\
\text { Prendedor }\end{array}$ & 4 & 200 & 1 & 32 \\
\hline & Ponta Rotativa & 4 & 400 & 2 & 140 \\
\hline \multirow[t]{3}{*}{ Desempenadeira } & $\begin{array}{c}2 \text { Rolos } \\
\text { Puxadores }\end{array}$ & 4 & 640 & 1 & 128 \\
\hline & $\begin{array}{c}5 \text { Rolos da } \\
\text { Mesa }\end{array}$ & 4 & 800 & 1 & 160 \\
\hline & 7 Cardans & 4 & 210 & 2 & 210 \\
\hline Carro de Bobinas & $\begin{array}{l}\text { Carro de } \\
\text { Bobinas }\end{array}$ & 4 & 100 & 1 & 100 \\
\hline \multirow{3}{*}{$\begin{array}{l}\text { Cadeira de } \\
\text { Laminação }\end{array}$} & Rolo Puxador & 4 & 260 & 1 & 100 \\
\hline & $\begin{array}{c}\text { Eixo } \\
\text { Intermediário }\end{array}$ & 4 & 200 & 1 & 64 \\
\hline & Cardans & 4 & 60 & 2 & 60 \\
\hline
\end{tabular}

A tabela 8 nos revela o tempo aproximado de aplicação de lubrificante em cada um dos conjuntos:

Tabela 8: Tempo médio de aplicação de lubrificante por conjunto

\begin{tabular}{|c|c|c|c|c|c|}
\hline \multicolumn{6}{|c|}{ Comparativo da quantidade de lubrificante utilizado nos conjunto em gramas } \\
\hline & & \multicolumn{2}{|c|}{ Antes } & \multicolumn{2}{|c|}{ Depois } \\
\hline & & $\begin{array}{l}\text { Frequência } \\
\text { Anual }\end{array}$ & Tempo & $\begin{array}{l}\text { Frequência } \\
\text { Anual }\end{array}$ & Tempo \\
\hline \multirow[t]{2}{*}{$\begin{array}{l}\text { Bobinadeira } \\
\text { Direita }\end{array}$} & $\begin{array}{c}\text { Rolo } \\
\text { Prendedor }\end{array}$ & 4 & 10 minutos & 2 & 10 minutos \\
\hline & Ponta Rotativa & 4 & 30 minutos & 4 & 30 minutos \\
\hline \multirow[t]{2}{*}{$\begin{array}{l}\text { Bobinadeira } \\
\text { Esquerda }\end{array}$} & $\begin{array}{c}\text { Rolo } \\
\text { Prendedor }\end{array}$ & 4 & 10 minutos & 2 & 10 minutos \\
\hline & Ponta Rotativa & 4 & 30 minutos & 4 & 30 minutos \\
\hline \multirow[t]{2}{*}{ Desbobinadeira } & $\begin{array}{c}\text { Rolo } \\
\text { Prendedor }\end{array}$ & 4 & 10 minutos & 1 & 10 minutos \\
\hline & Ponta Rotativa & 4 & 30 minutos & 2 & 30 minutos \\
\hline \multirow[t]{3}{*}{ Desempenadeira } & $\begin{array}{c}2 \text { Rolos } \\
\text { Puxadores }\end{array}$ & 4 & 40 minutos & 1 & 40 minutos \\
\hline & $\begin{array}{c}5 \text { Rolos da } \\
\text { Mesa }\end{array}$ & 4 & 70 minutos & 1 & 70 minutos \\
\hline & 7 Cardans & 4 & 40 minutos & 2 & 40 minutos \\
\hline Carro de Bobinas & $\begin{array}{l}\text { Carro de } \\
\text { Bobinas }\end{array}$ & 4 & 20 minutos & 1 & 20 minutos \\
\hline \multirow{3}{*}{$\begin{array}{l}\text { Cadeira de } \\
\text { Laminação }\end{array}$} & Rolo Puxador & 4 & 10 minutos & 1 & 10 minutos \\
\hline & $\begin{array}{c}\text { Eixo } \\
\text { Intermediário }\end{array}$ & 4 & 10 minutos & 1 & 10 minutos \\
\hline & Cardans & 4 & 10 minutos & 2 & 10 minutos \\
\hline
\end{tabular}

Com base no comparativo da tabela 7 e 8 , pode-se observar que atualmente seria utilizado cerca de 19800 gramas de lubrificante em um ano. Enquanto no plano de 
lubrificação proposta, objetiva se utilizar uma quantidade 4844 gramas de lubrificante em um ano, gerando uma economia de 14956 gramas de lubrificante.

No plano atual seria gasto cerca de 21 horas para a execução do trabalho, enquanto no plano proposto este tempo ira diminuir para 10 horas.

Considerando o preço do lubrificante $R \$ 5,5 \circ \mathrm{Kg}$, conseguimos uma economia anual de $R \$ 81,95$ reais de lubrificante. E considerando $R \$ 60$ reais o valor da mão de obra terceirizada conseguiu uma economia de 11 horas, o equivalente a $R \$ 660$ reis.

Economizando um total de $\mathrm{R} \$ 741$ reis anualmente com o laminador de bobinas.

\section{CONCLUSÃO}

A necessidade de se produzir mais e melhor, exige de que os segmentos industriais ajam de forma competitiva, procurando produzir com o máximo de eficiência possível. Para produzir mais, as empresas necessitam que seus equipamentos apresentem confiabilidade. A elaboração de um plano de lubrificação de qualidade, que apresente segurança ao equipamento, é um dos pilares, para que este mantenha-se operante de forma competitiva e sustentável em um cenário que já não mais permite desperdícios.

Espera-se que com a aplicação desta metodologia em todos os laminadores da linha de laminação a frio, se obtenha um significativo aumento da confiabilidade, além da diminuição do custo.

\section{Agradecimentos}

Gostaria de agradecer a Aperam South América, por ter viabilizado a execução deste trabalho, além de fornecer todos os recursos necessários para que o mesmo pudesse ser concluído com êxito.

\section{REFERÊNCIAS}

1 RIBEIRO, José Luis Duarte. Confiabilidade e Manutenção Industrial. São Paulo: Campus, 2009. 288 p.

2 ROUSSO, José. Manual de lubrificação industrial. São Paulo: Confederação Nacional da Indústria, 1982.

3 STOETERAU, R. L. Tribologia e Lubrificação. Universidade Federal de Santa Catarina, Santa Catarina, 2004.

4 GRUPO SKF. Catálogo de Rolamentos de Esferas - 2015. Cajamar, 2015. 1388 p.

5 ROLAMENTOS FAG LTDA. Rolamentos FAG - 1999. Santo Amargo. 720 p.

6 NEALE, Michael J. Tribology handbook. 2. ed. Boston: Planta Tree, 1995. 582 p. 